\title{
Increasing Cloud Usage: A Shift towards Green Clouds
}

\author{
Amritpal Kaur and Supriya Kinger \\ Department of Computer Science and Engineering \\ SGGS World University, \\ Fatehgarh Sahib, Punjab, India.
}

\begin{abstract}
As Cloud computing is being adopted widely and usage of internet services is increasing day by day, which gives rise to built large scale data centers. In cloud computing, a lot of research has been done on energy saving programs for reducing the energy consumption of large scale data centers. As large scale data centers consumes large amount of electrical power which affects the operating cost, performance as well as environment. This paper surveys the different energy saving techniques in network, protocols, algorithms and hardware infrastructure for energy- efficient operations.
\end{abstract}

\section{General Terms}

Cloud Computing, Service models, Green Cloud Computing and Energy saving techniques.

\section{Keywords}

Energy-efficiency, Para Virtualization, Full Virtualization, $\mathrm{co}_{2}$ footprints.

\section{INTRODUCTION}

Cloud Computing is the computing which uses the computer resources through internet. It is defined in [1],"A model for enabling convenient, on-demand network access to a shared pool of configurable computing resources that can be rapidly provisioned and released with minimal management effort or service provider interaction". Cloud computing is the delivery of Computing as a service rather than providing a product or software [3]. The principle of cloud computing is that companies and organizations do not longer manages and own their IT infrastructure, but it is delivered as a service by a Cloud Service Provider. Simple example of cloud computing is Yahoo email or Gmail etc. we don't need software or a server to using them. All a consumer would need is just an internet connection and we can start sending emails. Whereas mails are in use for several years and mail services have currently explored for providing network- based computing and network- based storage for end users, these services are called as "Cloud Computing" services [2]. The main advantage of cloud computing is that it delivers pay-as-you-go basis model i.e. Customers only pay for a service they use instead of paying for long-term licenses and/or investing in hardware which are not related to the actual usage.

Data center is the main component in cloud computing to store data and applications. It consists of tens of thousands of disks, network devices, servers and millions of users. So that's why significant amount of energy is consumed by large scale data centers [23]. According to estimation of Amazon, as its data centers, operating cost and operation of the servers report for 53\% of the total budget (based on a 3-year amortizationschedule), while energy-related costs amount to $42 \%$ of the total, and include both direct power consumption (approx.
$19 \%)$ and the cooling infrastructure (23\%) amortized over a 15 -year period [24]. So it has become very much necessary to focus on energy saving mechanisms in cloud computing in order to minimize the expenditure and also contributing towards the eco-friendly environment.

The objective of this paper is to give the brief introduction of Cloud Computing and Green Cloud Computing. Rest of the paper is organized as follows: section II discusses the service models of cloud computing, section III gives introduction to Green Cloud Computing and section IV describes the recent energy saving techniques in Green Cloud Computing and section $\mathrm{V}$ concludes the study.

\section{Service Models of Cloud}

Clouds are used for three main services i.e. software as a service, storage as a service and processing as a service [2]. This section discusses each of these cloud services.

\subsection{Software as a Service}

In this service, Cloud Service Provider provides the application or software for different consumers via internet. The software is managed in the cloud and all computation is executed in the cloud [2]. The consumer needs not to do the things such as purchasing the new version or updating the older one. In this way, consumer's cost is decreased. Software as a Service can also be called as "Software on Demand" because through Subscription, Provider gives the licenses of an application to customers as a Service according to their demands [23]. Example of software as a service is salesforce.com.

\subsection{Storage as a Service}

Consumer's primary data is stored in the cloud. Data may be of any type like audio video files, documents and images. Consumer can access the file from any location or any computer that has internet. No need to carry a physical storage device like pen-drives or CDs. The means of storing data in the cloud is called Data Centers [4]. The modification of file must be done at the consumer's PC and then upload the file back to the cloud. The cloud service provider ensures that there is enough space in the cloud [2]. Example of storage as a service is the Amazon Simple Storage Service.

\subsection{Processing as a Service}

Most of the jobs, which are not computationally demanding, are executed on the user's PC. Jobs which are computationally demanding uploaded and then processed in the cloud, results are returned to the user. Processing as a service provides the resources of a dominant server for definite large computational jobs. Example of processing as a service is the Amazon Elastic Compute Cloud service [2]. 


\section{Towards Green Cloud Computing}

Green Cloud Computing is the blend of energy efficient computing and cloud computing. Cloud computing is the rising technology that uses data centers to maintain the data. Information and communication technology is increased day by day. The industrial and academic infrastructure is being on cloud system. So, most of the organization and companies heading towards cloud computing to secure their relevant data, this raise to creation of large scale Data Centers. But, Data centers consumes enormous amount of electrical power which leads to energy consumption and increase in cost of data centers [5]. As the computing infrastructure consumes excess amount of power which results in emission of carbon dioxide $\left(\mathrm{CO}_{2}\right)$ and this leads to affects our environment. In order to reduce the energy consumption and $\mathrm{CO}_{2}$ emissions, it becomes necessary to develop the energy saving mechanisms for efficient usage of resources in cloud computing.

\section{Energy-Saving Techniques}

Now days, Cloud Computing has been focusing on approach of energy saving techniques for delivering ICT services and this can be done if some improvements has been done regarding utilization of data center resources [6]. EnergySaving techniques in Green Cloud Computing can be energy efficient hardware, Dynamic Voltage and Frequency Scaling (DVFS), Terminal servers and thin clients, Virtualization of resources and energy-efficient network and protocols. In the following sections each of the technique is going to be discussed.

\subsection{Energy Efficient Hardware}

In order to save the energy it has become necessary to develop energy efficient hardware. US Energy Star has initiated the step regarding manufacturing IT products which are not only being worth energy saving but also being beneficiary for our environment [7].

\subsubsection{Energy Efficient Processors}

Processors plays vital role in the data center as it consists of several physical machines. Current processors have been considered to offer not only high performance but also worldclass efficiency. This is due to high energy consumption which not only translates to high power bills for maintenance, but also results in high heat dissipation which decreases the reliability of hardware [31]. The newly discovered techniques like SpeedStep [8], PowerNow [9], Cool'nQuiet [10] and Demand- Based Switching [11] helps in power consumption of processors. When the machine is idle, the above mechanisms power off the parts of chip and slow down the speed of CPU clock [12, 13]. The recent technologies like solid state discs which consumes less energy as compared to present technology being used for hard disc drives [6].

The usage of energy-efficient monitors like $\mathrm{LED}_{\mathrm{s}}$ rather than using CRT monitors this result into less consumption of energy. In addition, the unnecessary printing must be avoided in order to reduce the usage of printers, because while printing carbon emissions are released.

\subsubsection{Memory}

The contribution towards the noteworthy fraction of the power budget and with the progression of ICT, there is explosively growth in requirement of storage devices. With the need of more space, the operating cost gets higher due to lower density of servers and then it is required to develop energy saving techniques for memory [31]. In a data center durable storage of data is provided by hard disk arrays. Hard disk provides functionality such as disk array controllers, disk enclosures, cache memories and redundant power supplies [2]. In the data center all the storage is consolidated and hard disk usage is centrally coordinated [25, 26]. Thus, increase the energy efficiency of storage. Some of the files are not accessed regularly are stored in different set of capacity optimized hard disks [27]. These hard disks, which are not in use enables low-power mode and consumes no energy. Techniques for conserving storage energy have been comprehensively explored for more than ten years, and the research object mainly includes a single-disk-based mobile device (e.g., laptop) and a multiple-disks-based storage system in a data center [28].

\subsection{Dynamic Voltage Frequency Scaling (DVFS)}

DVFS is a technique which helps not only in lowering the operating voltage and frequency but also considerably helps in decreasing power consumption of the resources. Modern processors are in built by DVFS technique which enables to operate the processors at several frequencies under different supply of voltage [14].Now days this technique is used in laptop systems in order to lower the consumption of battery power. The power consumption is reduced when the clock speed is lowered and when there is reduction in voltage during idle time and intensive application phases [14]. For example, DVFS technique is used by large data centers in Cluster computing and Supercomputing in order to attain high reliability and to make reduction in power consumption $[15$, 16]. This technique is used in latest $\mathrm{CPU}_{\mathrm{s}}$ such as Intel's Speed and AMD's Power Now! technologies and these lowers the CPU voltage and Frequency [17].

\subsection{Terminal Servers and Thin Clients}

A terminal server is the hardware device which provides resources like printers, desktops, laptops and other peripheral devices having a connection of common point with LAN and WAN [22]. Thin client is that model by which we can achieve the target of utilization of computing resources of terminal servers. In this technique the computing resources are utilized and shared by the multiple users. With the usage of this model, the clients can converts user inputs to the server and in exchange of this the server provides update to the client [18]. It contributes toward lower and better utilization of the hardware thus reduces the power consumption. Fig. 1 shows the brief description of Terminal Servers and Thin Clients

\subsection{Virtualization}

Virtualization is the current technology by which energy efficient operations can be applied in data centers [6]. It allows multiple operating systems to be executed simultaneously on the same physical machine, therefore reduces the number of servers in data centers and resource are 


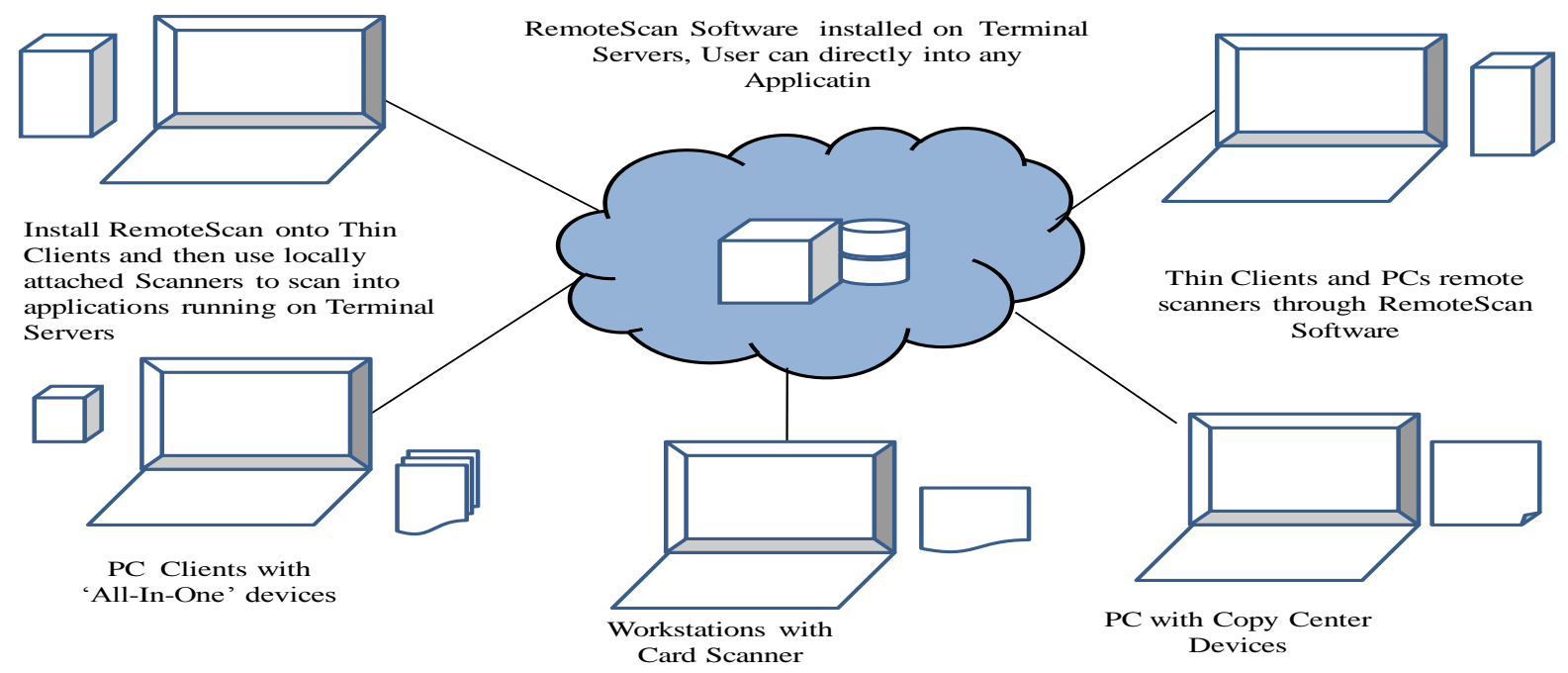

Fig 1. Terminal Servers and Thin Clients [29]

Utilized efficiently. The most popular virtualization technology solution providers are VMware and KVM [21]. There are two main different techniques to perform Virtualization i.e. full virtualization and paravirtualization. Fig 2 shows the architecture of Virtualization.

\subsubsection{Full Virtualization \& Para Virtualization}

Full Virtualization is that platform in which the operating system like kernel known as hypervisors, and this consists of Virtual Machine Monitor (VMM) and kernel component. The hypervisor keeps each virtual server completely independent and it is unaware of the other virtual servers. Each guest server runs on its own operating system [19]. Besides this, there is no host operating system. This usually works with the management and they run on the top of the kernel. Full virtualization helps in giving the best security and isolation for machines and it simply helps in doing portability and migration of virtual machines. Examples of full virtualization are VMware's virtualization products and Microsoft virtual server [20].

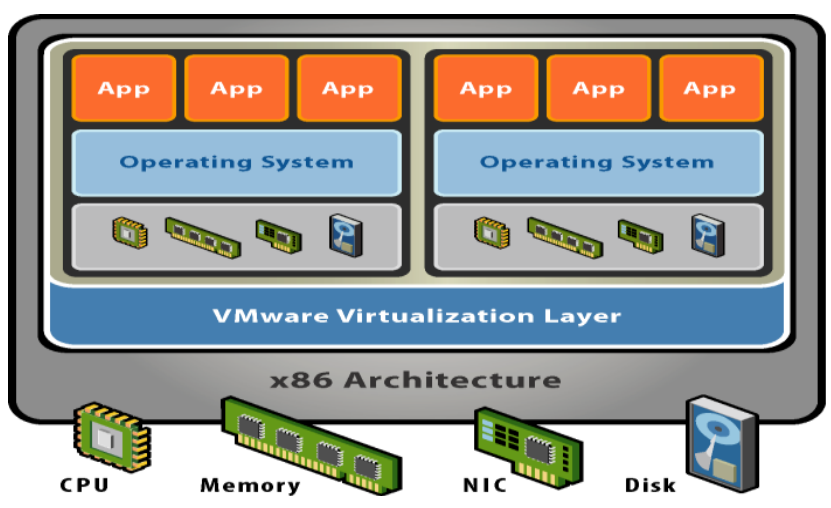

Fig. 2 Architecture of Virtualization [20]

On the other hand, the paravirtualization is different from full virtualization. As paravirtualization interacts with the guest
Operating system and also does the modification in order to improve performance and efficiency. Paravirtualization hypervisor does not need much processing power in order to manage guest operating system because each system is being aware of demand of other operating system. In present, Para virtualization provides support only to open source operating systems and this bound its applicability.

\subsection{Energy Savings in Network and Protocols}

One of the largest consumers of energy is communication technology, however energy optimization for communication must deal with the trade-offs between performance, energy savings and QoS [30]. Network devices consume enamours amount of power as table 1 lists the energy consumed in corporate network and the internet IP network as well as the power consumption and capacity of the network devices [2].

Table 1. Equipment in Model of network [2]

\begin{tabular}{|c|c|c|c|}
\hline & Equipment & Capacity & $\begin{array}{c}\text { Power } \\
\text { Consumption }\end{array}$ \\
\hline Storage & $\begin{array}{l}\text { HP } 8100 \\
\text { EVA }\end{array}$ & $604.8 \mathrm{~Tb}$ & $4.9 \mathrm{~kW}$ \\
\hline $\begin{array}{l}\text { Content } \\
\text { Server }\end{array}$ & $\begin{array}{l}\text { HP DL380 } \\
\text { G5 }\end{array}$ & $800 \mathrm{Mb} / \mathrm{s}$ & $225 \mathrm{~W}$ \\
\hline $\begin{array}{c}\text { Computation } \\
\text { Server }\end{array}$ & $\begin{array}{l}\text { HP DL380 } \\
\text { G5 }\end{array}$ & & $355 \mathrm{~W}$ \\
\hline LAN & Cisco 6509 & $320 \mathrm{~Gb} / \mathrm{s}$ & $3.8 \mathrm{~kW}$ \\
\hline $\begin{array}{l}\text { Gateway } \\
\text { Router }\end{array}$ & $\begin{array}{l}\text { Juniper } \\
\text { MX-960 }\end{array}$ & $660 \mathrm{~Gb} / \mathrm{s}$ & $5.1 \mathrm{~kW}$ \\
\hline
\end{tabular}


Network optimization must be considered in which virtual machines inter communicating for optimization of virtual network topologies. There should be network communication between virtual machines considered in reallocation decisions in order to reduce the data transfer overhead and network devices load [5]. Network protocols can also be optimized and redeveloped in a manner that improves the energy-efficient operation of network elements. Network devices must transfer services to device which is more efficient device that needs to be always on, while certain other devices are turned off. It is impossible to turn off certain system components because of 'soft states' so that's why new protocols need to be proposed to deal with soft states so as to increase the energy efficiency of the network [6]. In addition, network devices can be utilized by three main techniques i.e. Network Traffic Consolidation (NTC), Server Load Consolidation (SLC) and Link State Adaptation (LSA) [31].

\section{Conclusion}

In this paper, the basic concepts of Cloud Computing and its core services have been discussed. Then, a survey of different energy saving techniques for efficient usage of resources has been done in this paper. Due to a drastic shift towards cloud computing, there is increase in environmental hazards like increasing carbon footprints, etc. So there is a need for energy efficient resource scheduling and management. Resource scheduling in Green Clouds can be either load based or can be temperature based. Our future work will explore a scheduling system that is both power-aware and thermal-aware simultaneously, to maximize energy savings both from physical servers and the cooling systems used.

\section{REFERENCES}

[1] P. Mell, and T. Grance, "The NIST Definition of Cloud computing", National Institute of Standards and Technology, 2009.

[2] Jayant Baliga, Robert W. A. Ayre, Kerry Hinton, and Rodney S. Tucker, "Green Cloud Computing: Balancing Energy in Processing, Storage, and Transport", in the proceedings of the 2011 IEEE, Vol. 99, No. 1, pp.149167.

[3] Peeyush Mather and Nikhil Nishchal. 2010. Cloud Computing: New challenge to the entire computer industry. In the Ist International Conference on Parallel, Distributed and Grid Computing, pp.223-225.

[4] Jiyi WU, Lingdi PING, Xiaoping GE, Ya Wang and Jianqing FU. 2010. Cloud Storage as the Infrastructure of Cloud Computing. In the International Conference on Intelligent Computing and Cognitive Informatics, pp.380-383.

[5] Anton Beloglazov and Rajkumar Buyya. 2010. Energy Efficient Resource Management in Virtualized Cloud Data Centers. In the IEEE/ACM International Conference on Cluster, Cloud and Grid Computing, pp.826-829.

[6] A. Berl, E. Gelenbe, M. D. Girolamo, G. Giuliani, H. D. Meer, M. Q. Dang and K.Pentikousis, "Energy-Efficient Cloud Computing", The Computer Journal, Vol. 53, No. 7, 2009, in Oxford University Press on behalf of The British Computer Society.

[7] Energy Star, http://www.energystar.gov.
[8] Intel, White Paper, "Wireless Intel SpeedStep Power Manager: optimizing power consumption for the Intel PXA27x processor family", 2004.

[9] AMDPowerNow!Technology,http://www.amd.com/dede /Processors/ProductInformation/0,30_118_10220_10221 964,00.html.

[10] AMDCool'n'QuietTechnology,http://www.amd.com/ded e/Processors/ProductInformation

[11] Intel Software Network (2008) Enhanced Intel SpeedStep Technology and Demand-Based Switching on Linux.http://softwarecommunity.intel.com/articles/eng/1 611.htm

[12] "ENERGY STAR* system implementation", Intel with technical collaboration from the U.S.Environmental ProtectionAgency, White Paper-001, 2007.

[13] C.Windeck. 2007. Energy Star 4.0. c't Magazin für Computer Technik", pp.52-53.

[14] Lizhe Wangy, Gregor von Laszewskiy, Jai Dayalz and Fugang Wangy. 2010. Towards Energy Aware Scheduling for Precedence Constrained Parallel Tasks in a Cluster with DVFS. IN the 10th IEEE/ACM International Conference on Cluster, Cloud and Grid Computing, pp.368-374.

[15] G. Chen, K. Malkowski, M. Kandemir, and P. Raghavan. 2005. Reducing power with performance constraints for parallel sparse applications. In Proceedings of the 19th IEEE International Parallel and Distributed Processing Symposium (IPDPS'05) - Workshop 11. Washington, DC, USA: IEEE Computer Society, pp. 231.

[16] R. Ge, F. X., F. W., and K. Cameron. 2007. CPU MISER: A Performance-Directed, Run-Time System for Power-Aware Clusters. In Proceedings of the 2007 International Conference on Parallel Processing, IEEE Computer Society Washington, DC, USA.

[17] Rolf Harms and Michael Yamartino, "The Economics of the Cloud", Microsoft, White paper, 2010.

[18] Jason Nieh, S. Jae Yang and Naomi Novik. 2000 A Comparison of Thin-Client Computing Architectures", Technical Report CUCS-022-00, Columbia University.

[19] http://computer.howstuffworks.com/servervirtualization2 .htm.

[20] VMware, "Understanding Full Virtualization, Paravirtualization, and Hardware Assist", White Paper.

[21] J. Stoess, C. Lang, and F. Bellosa. 2007. Energy Management for Hypervisor-Based Virtual Machines. USENIX Annual Technical Conference, pp.1-14.

[22] http://searhiomidmarket.techtarget.com/definite/terminal _server.

[23] Muzafar Ahmad Bhat, Razeef Mohd Shah, Bashir Ahmad and Inayat Rasool Bhat, "Cloud Computing: A Solution to Information Support Systems (ISS)" International Journel of Computer Aplications, 2010.

[24] J Hamilton. 2009. Cooperative expendable micro-slice servers (CEMS): low cost, low power servers for internet-scale services. In Proceedings of CIDR, California, USA. 
[25] M. Armbrust, A. Fox, R. Griffith, A. D. Joseph, R. H. Katz, A. Konwinski, G. Lee, D. A. Patterson, A. Rabkin, I. Stoica, andM. Zaharia. 2009 Above the clouds: A Berkeley view of cloud computing", Tech. Report. UCB/EECS-2009-28.

[26] F. Chang, J. Dean, S. Ghemawat, W. C. Hsieh,D. A. Wallach, M. Burrows, T. Chandra, A. Fikes, and R. E. Gruber. 2006. BBigtable: A distributed storage system for structured data. In Proceeding of $7^{\text {th }}$ Symposium Operating System Design Implementation, pp.205-218.

[27] D. Colarelli and D. Grunwald. 2002. Massive arrays of idle disks for storage archives. In Proceeding of ACM/IEEE Conference on Supercomputures, pp.1-11.
[28] Xue-Liang Liao, Shi Bai, Yu-Ping Wang andShi-Min Hu. 2011. ISRA-Based Grouping: A Disk Reorganization Approach for Disk Energy Conservation and Disk Performance Enhancement. IEEE Transactions on Computers, vol. 60, issue no. 2, 2011, pp.292-304.

[29] http://www.remotescan.com/terminalservicediagram.php.

[30] E. Gelenbe and S. Silvestri. 2009. Reducing Power Consumption in Wired Networks. In Proceeding of 24th Annual International Symposium on Computer and Information Sciences (ISCIS), pp.717-729.

[31] Si-Yuan Jing, Shahzad Ali, Kun She and Yi Zhong 2011. State-of-the-art research study for green cloud computing. Springer Science and Business Media. 